\title{
What 5G has been and what should 5G+ be?
}

\author{
By Zoran Bojkovic ${ }^{*}$, Dragorad Milovanovic ${ }^{ \pm}$, Tulsi Pawan Fowdur ${ }^{ \pm}$\& \\ Mussawir Ahmad Hosany ${ }^{+}$
}

In this work we aim to provide a comprehensive summary of the most inspiring aspects of $5 G$ mobile networks. In addition, we present the latest findings on the promising $6 G$ technology towards a world of fully digital connectivity. The key determinants are extreme system performance and combinations of requirements for new use cases. We study new performance targets beyond $5 G$ in two stages: $5 G+$ evolution and new $6 G$ step. Relevant technologies considered too immature for $5 G$ or outside the defined scope are outlined. To justify our vision of future mobile networks, we point out the need for closer collaborations of academia, standardizing bodies, industrial organizations and governments. The contribution strategy is gradual evolution and performance enhancement of mobile communications. Finally, we identify challenges and directions on network technology roadmap toward $6 G$.

Keywords: wireless communication, mobile broadband, low-latency communication, $5 G$ network, $3 G P P, 6 G$ vision

\section{Introduction}

The new $5 \mathrm{G}$ generation of mobile telecommunications systems meet the ITUR IMT-2020 (International Mobile Telecommunications-2020) requirements by supporting the Internet Protocol (IP) network with higher data rates, larger number of connections, and considerable lower latency than previous generations (Bojkovic et al. 2020, Milovanovic et al. 2019, Milovanovic and Bojkovic 2019). Over the past years, the first complete set of global technical standards has enabled a basic commercial deployment of $5 \mathrm{G}$ mobile networks. Consumers and businesses are looking to a $5 \mathrm{G}$ wireless connection to enable faster downlink/ uplink speeds and high quality of service (QoS) that's secure and reliable in new vertical applications of Smart transportation ITS, industrial IoT, eHealth, Smart cities. The academic community as well as industrial organizations are already turning their considerations towards the next generation of mobile communication termed as 6G (Andrews et al. 2014, Tripathi and Reed 2020, David and Berndt 2018).

With a view of achieving enhanced performance of $5 \mathrm{G}+$ core services we identified three methods in this work. Mobile broad bandwidth and low latency (mBBLL) service will provide a high-quality experience (QoE) in typical

\footnotetext{
"Professor, University of Belgrade, Serbia

${ }^{ \pm}$Lecturer, University of Belgrade, Serbia.

"University of Mauritius, Mauritius.

${ }^{+}$University of Mauritius, Mauritius.
} 
applications of mobile augmented/virtual reality (AR/VR) and holographic teleconferencing. Next, massive broadband width machine type (mBBMT) service enhances 5G mobile broadband and machine communication in typical application of tactile IoT. And finally, massive low-latency machine type (mLLMT) service enhances ultra-reliable and low-latency communication in typical application of large scale industrial IoT (Dang et al. 2020, Saad et al. 2020).

Objective of the paper is to review evolution of mobile network starts from the first $1 \mathrm{G}$ to newest $5 \mathrm{G}$ generation. Next, the main objective in collaborations of industry experts, academia and international organizations is to identify challenges and directions on network technology roadmap toward 6G. We study new performance targets beyond $5 \mathrm{G}$ in two stages: $5 \mathrm{G}+$ evolution and new $6 \mathrm{G}$ step. The key determinants are extreme system performance and combinations of requirements for new use cases in terms of functionalities and quality of experience. Paving the way towards 6G, relevant technologies considered too immature for $5 \mathrm{G}$ or which are outside the defined scope will be included. Finally, we study three technology milestones for the upcoming years.

\section{Background}

Every one to two years there are new 3rd Generation Partnership Project (3GPP) releases leading to a constant evolution of cellular communication systems. A new generation is derived from one of the releases in general every 10 years or decade. Technology research at the conceptual level along with a vision of a typical use case and deployment scenario is brought about at the start of every 10 year cycle. The ultimate goal of commercial network deployment is achieved by following established research procedures, development of specifications and finally prototyping of the systems. It is expected that significant improvements will be achieved with the design of a new generation standard. The evolution of mobile network starts with the first analog mobile standards termed as $1 \mathrm{G}$, then by the second generation (2G) standard termed as GSM (Global System for Mobile Telecomunications), followed by UMTS (Universal Mobile Telecommunication Systems) which is the technology for the third generation (3G), then LTE (LongTerm Evolution) which makes up the fourth generation (4G), and finally the current generation which is $5 \mathrm{G}$ as shown in Figure 1. The future or post $5 \mathrm{G}$ generation is termed as $6 \mathrm{G}$. The implementation of $6 \mathrm{G}$ systems expected to start between 2027 and 2030. Larger spectral and energy efficiencies as well as data rates, lesser latency and improved security, as well as enhanced quality of service (QoS) are some basic issues that need to be addressed in beyond $5 \mathrm{G}+$, system to make the transition from 5G. Toward 6G systems, all the potential key performance indicators (KPIs) must be attained by proceeding from the evolution of $5 \mathrm{G}$ systems. $6 \mathrm{G}$ communication is still in its infancy and research activities on 6G are in their initial stages. Several studies will be initiated globally on the standardization of 6G as from 2020 (ITU-T 2019, Posio 2020, 3GPP Technical Specification Group Services and System Aspects 2020). 
Figure 1. Development of Data Rate and Application of Mobile Wireless Networks through Decades from 1980 to 2030 Years

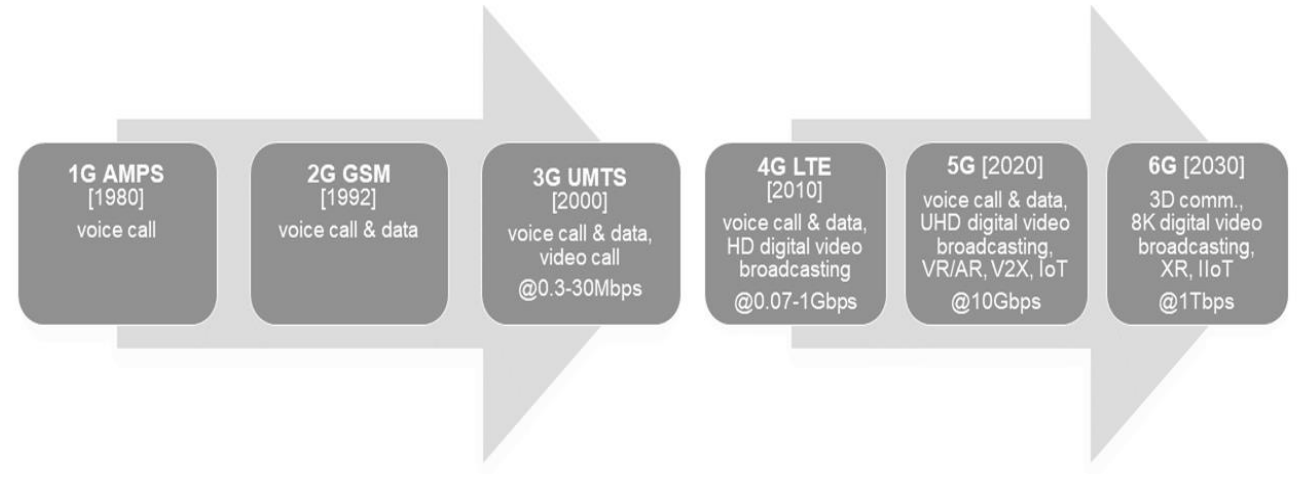

Increased in spectral and energy efficiencies as well as network reliability and reduction in communication delays are all the goals set for a successful 5G communication network. It employs the microwave spectrum in the range 3.3$4.3 \mathrm{GHz}$ as well as the mmWave band increasing in such a manner data rates up to 10 Gbps. It should be noted that BDMA (Beam division multiple access) and FBMC (Filter bank multicarrier) are used, together with many technologies integrated into $5 \mathrm{G}$ with the final objective to advance the characteristics of a network. The following $5 \mathrm{G}$ components have been implemented with a view to enhance user experience: Massive MIMO (multiple-input and multiple-output) systems to increase spectral efficiency, Software defined networks (SDN) to provide flexible connections, information content networking (ICN) to reduce network traffic and network slicing for fast deployment.

$5 \mathrm{G}$ proposes three major scenarios in its communication domain and these are enhanced mobile broadband (eMBB), ultra-reliable and low latency communications (uRLLC) and massive type communications (mMTC). In order to enable various types of communication links, there are many protocols that have been implemented in the 5G standard. There are two reasons for this: the first one is supply, because same techniques still require experimental verification as well as test in practical situation. Secondly, from the demand point of view, advanced communication technologies employing different services and devices, despite that are not fully recognized as being important. Since it is difficult to find solution for all related problems, $5 \mathrm{G}$ is presently viewed as just an initial phase of deployment in the process of interconnection of user devices. Since limitation of radio coverage in service areas does not exist in 5G global standard, all user devices can be interconnected from any part of the globe. Therefore, research beyond $5 \mathrm{G}$ will aim to remove the differences between the definition and realization surpassing the existing vision and requirements (Posio 2020).

Presently the deployment of 5G standards for cellular networks is being considered and both the academic community as well industry are turning their attention to what comes next. In practice, it takes around ten years to design, implement and deploy a novel cellular networking standard. There this is the perfect time to research promising areas and novel directions for the next decade thereby laying the foundations for a possible $6 \mathrm{G}$ communication standard for 
cellular networks. In transition to 5G, the main innovation from the physical layer is simultaneously support for variety of applications and services with different system data rates, delays and reliability requirements. This novelty has been possible by the OFDM (orthogonal frequency-division multiplexing) technology as well as by the concept of network slicing and massive MIMO systems that make use of very large antenna arrays at the base stations (Tripathi and Reed 2020).

\section{G+ Technology Roadmap}

In order to design, implement and deploy the 5G technology it is imperative to have the collaborations among various industry experts, academia and international organizations that regulate standards. The main objective of such collaborations is to identify challenges and directions for cellular network technologies thereby, flagging the way for providing massive connectivity for wireless communications. Software defined (SDN) and network function virtualization (NFV) frameworks have to be with distributed systems software to enable all 5G+ systems. Other hand of systems are end-user integration, security, spectral and energy co-efficiency, maintaining service quality, faults tolerance of any kind. Some drastic upgrade of the whole communication needs to happen by this year 2020, in order to support the forecasted growth. It is imperative that industry for future networks participate in $5 \mathrm{G}$ and beyond technology roadmap activity.

The 3rd Generation Partnership Project (3GPP) is a consortium of standards organizations with technology roadmap of protocols for mobile telecommunications. 3GPP system provides Releases with a stable platform to researchers and developers with a view to implement features at a given instant and then allow for the addition of new functionality in subsequent Releases. The TR 21.900 Release provides mechanisms create and maintain specifications. 5G implementation so far (Release 15) are for public consumer type services. 5G Release 16 with focus on industry vertical being finalized only now (June 2020 with 3-month delay from March 2020). 5G Release 17 is scheduled for freeze Sep. 2021 and delivery in Dec. 2021. Work on 5G Release 18 starts in Sept. 2020. 6G Release 20 is expected around 2025 (3GPP Technical Specification Group Services and System Aspects 2020).

- Phase 1 (3GPP Release 15 TR21.915) work began in June 2016 and was completed in September 2018. The NR sub system is presently being deployes commercially and it focuses on the eMBB use case.

- Phase 2 (3GPP Release 16 TR21.916) is a major release for the project, because it will bring the specification organization's IMT-2020 RIT/SRIT submission (to ITU-R WP 5D) for a complete 3GPP 5G system. Release 16 has been put on hold in March 2020 with a targeted date of June 2020, when complete 5G standards are published.

- Phase 3 (3GPP Release 17 TR21.917) focuses mainly on the improvements of a 5G+ system which already started in December 2019 and scheduled 
for delivery in Dec. 2022. Regarding the architectural system of 5G, 3GPP provides Release 17 and beyond which has as objective to enhance support of IloT (Industrial internet of Things) and convergence from wireless or wired users. Also, the Release 17 and beyond will have to improve support for multicast and broadcast architecture, proximity services, enhance provision of multi-access edge computing, and enhance care of network automation. With regard to the Radio Access Network (RAN) the community of 3GPP, in June 2019, identified many core research areas to be considered in Release 17. These areas are given as follows:

1. NR-Light: To enable lightweight communications for industrial sensors and similar applications.

2. IIoT and MIMO improvements.

3. Sidelink enhancements for both V2X (Vehicle-to-everything) and public safety.

4. Provision and support to enhance coverage.

5. Extension of $5 \mathrm{G}$ NR to operate in frequencies beyond $52 \mathrm{GHz}$ to be included in Release 18. Deadline for Release 18 Stage 1 not yet decided, possibly December 2020 or later.

The 5G has been deployed with a view to provide drastic increase of wireless data traffic and support of other usage scenarios. Also, 5G+ and 6G technologies are anticipated to lengthen $5 \mathrm{G}$ competencies. There are many strict requirements that need to be met for providing high figures of merit such as increased bit rates (up to Tbps) and lower delays - such as pervasive edge intelligence, ultra-massive machine-type communication, holographic rendering and high precision communications. These strict requirements take the dimension of energy efficiency, intelligence, spectral efficiency, security, secrecy and privacy. Machine learning techniques such as deep and reinforcement learning are applied in the 5G technology as specific artificial intelligence approaches with a view to plan, schedule, represent knowledge and provide optimization. This enables to enhance user experiences and optimize networks more efficiently as well as provide high quality software applications. For instance, there are many data-demanding applications such as massive as well as Industrial IoT (IIoT), automatic robotic platforms including control, sensors and actuators, as well as the integration to other techniques into cyber-physical systems will impose new performance requirements (Dang et al. 2020).

\section{Performance Indicators towards 6G}

$5 \mathrm{G}+$ will be implemented with the main objective of reducing latency among user devices and enhancing reliability for services that extend beyond edge cloud or private environments. This objective is presently inspiring because of the absence of an advanced transport network protocol architecture. Heterogeneous network segments together with edge fabric may also integrate the 5G+ 
environment and this will facilitate configuration and control of new applications and services. It is envisaged that $5 \mathrm{G}+$ will provide data rates in excess of hundreds of Mbps with delays of less than $1 \mathrm{~ms}$ while simultaneously assuming connections to billions of devices. In order to radically change the living and working styles of mobile users services such as entertainment, e-Health, Industrial IoT, smart cities and smart transportation have to be incorporated in the verticals of $5 \mathrm{G}+$.

The $6 \mathrm{G}$ technology is expected to provide much wider application scenarios than what $5 \mathrm{G}+$ will do. It is important to have backward compatibility with the $5 \mathrm{G}$ primary services such as eMBB, mMTC and uRLLC. $6 \mathrm{G}$ will also include novel services such as holographic communication, personal monitoring, drone taxi, Internet of robots, etc. One of the most significant applications is virtual and augmented reality (AR/VR). On the other hand, holographic communication (HC) is considered as a high throughput requirement of $6 \mathrm{G}$. In the near future, media interaction will be transformed from the current plane multimedia to high-fidelity $\mathrm{AR} / \mathrm{VR}$ and to wireless holographic communication.

As shown in Table 1, new performance targets beyond $5 \mathrm{G}$ will be met in two stages: $5 \mathrm{G}+$ evolution, and new $6 \mathrm{G}$ step.

Table 1. Performance Indicators $5 G$ vs. $5 G+v s .6 G$

\begin{tabular}{|l|c|c|c|}
\hline Performance measure & 5G & 5G+ & 6G \\
\hline Peak data rata & $10-20 \mathrm{Gbps}$ & $100 \mathrm{Gbps}$ & $1 \mathrm{Tbps}$ \\
\hline Per-user data rate & $1 \mathrm{Gbps}$ & $10 \mathrm{Gbps}$ & $>10 \mathrm{Gbps}$ \\
\hline Traffic density & $10 \mathrm{~Tb} / \mathrm{sm}^{2}$ & & $>100 \mathrm{~Tb} / \mathrm{sm}^{2} / \mathrm{km}^{2}$ \\
\hline Connection density & $10^{6} / \mathrm{km}^{2}$ & & $10 / \mathrm{km}^{2}$ \\
\hline Delay & $\mathrm{ms} \mathrm{level}$ & $1 \mathrm{~ms}$ & $<1 \mathrm{~ms}$ \\
\hline Mobility & $350 \mathrm{~km} / \mathrm{h}$ & & $>1000 \mathrm{~km} / \mathrm{h}$ \\
\hline Spectrum efficiency & $3-5 x$ relative to $4 \mathrm{G}$ & & $>3 x$ relative to $5 \mathrm{G}$ \\
\hline Energy efficiency & $1000 x$ relative to $4 \mathrm{G}$ & & $>10 x$ relative to $5 \mathrm{G}$ \\
\hline Coverage percent & $70 \%$ & $80 \%$ & $>90 \%$ \\
\hline Reliability & $99.9 \%$ & $99.99 \%$ & $>99.999 \%$ \\
\hline
\end{tabular}

Source: Dang et al. 2020.

The energy efficiency, density and data rate is projected to increase tenfold in 6G. Spectrum efficiency and mobility are expected to be enhanced threefold. Moreover, the latency in $6 \mathrm{G}$ will be brought down further to below $5 \mathrm{~ms}$. Rise from the current 70 percent to 99 percent is expected for the coverage percentage. Presently the reliability in $5 \mathrm{G}$ is $99.9 \%$ and this will increase to $99.999 \%$ in $6 \mathrm{G}$. Positioning error will be reduced from the current meter range to the centimeter range.

The key $6 \mathrm{G}$ determinants are extreme system performance and combinations of requirements for new use cases (Figure 2, Table 2). 
Figure 2. Evolution of $5 G$ Primary Service Classes to Extreme 6G Performances for Specific Use Cases and Combinations of Requirements for New Use Cases
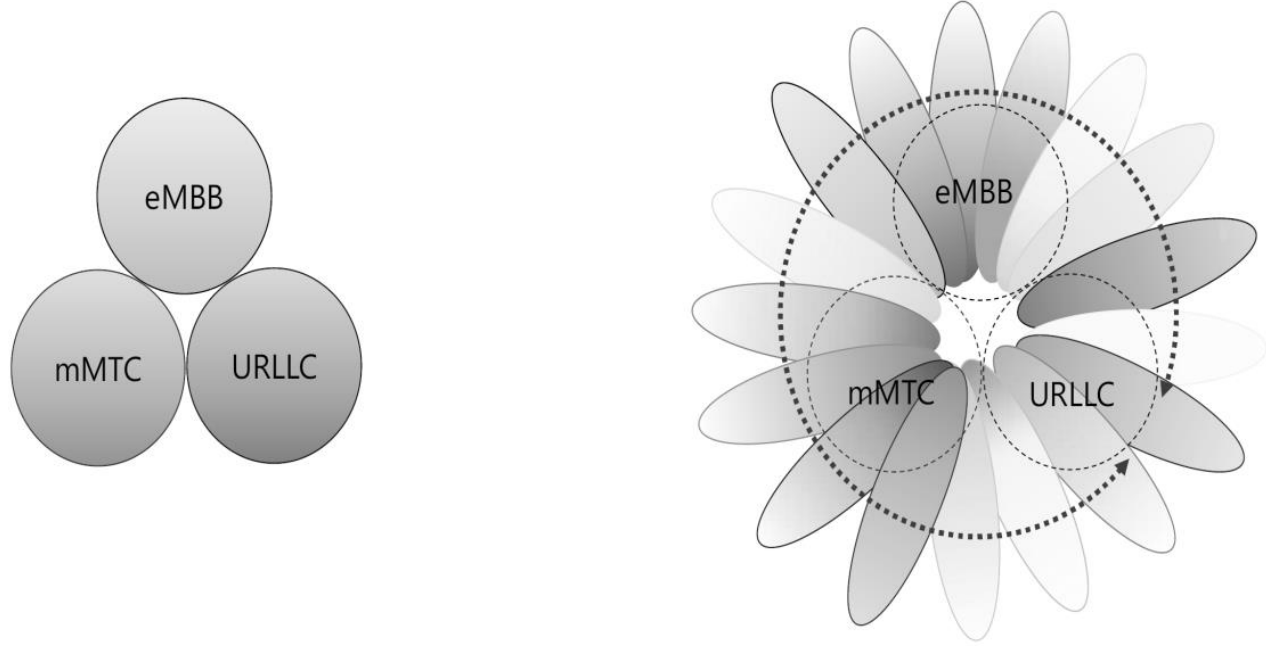

Table 2. Application Types $5 G$ vs. $5 G+v$ s. $6 G$

\begin{tabular}{|l|c|c|c|}
\hline & $\mathbf{5 G}$ & $\mathbf{5 G +}$ & $\mathbf{6 G}$ \\
\hline Applications & eMBB & Reliable eMBB & MBRLLC \\
& URLLS & URLLC & mURLLC \\
& mMTC & mMTC & HCS (human-centric services) \\
& & Hybrid & MPS (multi-purpose services) \\
& & (URLLC+eMBB) & \\
\hline
\end{tabular}

Source: Tripathi and Reed 2020.

Most of the use cases in $6 \mathrm{G}$ are evolving from the emerging $5 \mathrm{G}$ system based applications in terms of functionalities and quality of experience. However, 6G new use cases will combine experience sharing AR/VR and telepresence applications, increasing per-user data rate from $100 \mathrm{Mbps}$ to $1 \mathrm{Gbps}$, peak data rate from $20 \mathrm{Gbps}$ to $1 \mathrm{Tbps}$, and decreasing air-interface latency from $1 \mathrm{~ms}$ to 100 micros. Remote control applications of eHealth, Industry 4.0 and unmanned mobility will be combined in new use cases with reliability increase from $99.999 \%$ to $99.99999 \%$, spectrum efficiency $5 \mathrm{x}$ from $5 \mathrm{G}$, as well as vehicle speed from $500 \mathrm{kmph}$ to $1000 \mathrm{kmph}$. Connecting everything applications of pervasive connectivity with increase spectrum efficiency $5 \mathrm{x}$ from $5 \mathrm{G}$, per-user data from $100 \mathrm{Mbps}$ to $1 \mathrm{Gbps}$, number devices from $10^{6} \mathrm{pkm}^{2}$ to $10^{7} \mathrm{pkm}^{2}$, as well as energy efficiency 10-100x from 5G.

\section{Paving the Way towards 6G}

The immature features that $5 \mathrm{G}$ could not support will now be included in $6 \mathrm{G}$ with the relevant technologies. Focusing on the physical layer of a 6G technology one can find many research opportunities in order to increase further the data rates offered by the $5 \mathrm{G}$. For example, using carrier frequencies above $40 \mathrm{GHz}$, including even the terahertz bands. The high carrier frequency band will increase the spectral 
channels with bandwidths of several gigahertz to tens of $\mathrm{GHz}$ possible. Another opportunity that $6 \mathrm{G}$ can be accommodated is the provision of low-resolution multiple antenna architectures with digital beamforming to support multiple users thereby providing higher flexibility than what was provided by $5 \mathrm{G}$. The third opportunity is to re-investigate large bandwidths in the context of signal processing models. Robotics is a characteristic use case of signal processing and will be found in the factory in the future. Another key research direction for $6 \mathrm{G}$ is machine-learning toolset. It has been already envisaged that $6 \mathrm{G}$ will be implemented with Artificial Intelligence algorithms at all levels. The departure point in the implementation process would be from the network orchestration and management to coding and signal processing in the physical layer. Furthermore, in the $6 \mathrm{G}$ implementation there would be the manipulation of smart structures and data mining in the network as well as device level for service-based communications. A 6G driving trends and enabling technologies are shown in Table 3.

Table 3. $6 G$ Driven by Innovative Applications and Enabling Technologies

\begin{tabular}{|lll|}
\hline Driving applications & Driving trends & Enabling technologies \\
Combine experience & More bits, spectrum, & Radio spectrum above 6 GHz \\
sharing (multisensory & reliability & Transceivers with integrated \\
XR, telepresence) & Spectral and energy & frequency bands \\
Remote control and & efficiency & Integrate terrestrial, airborne \\
autonomous systems & From areal to volumetric & and satellite networks \\
Connecting & Convergence of & Energy transfer and \\
everything & communication, sensing, & harvesting \\
& control and computing & Emergence of smart surfaces \\
& & and environments \\
\hline
\end{tabular}

Due to the unprecedented increase in the number of innovative applications it is imperative to have a new cellular generation. $6 \mathrm{G}$ is no exception: In fact, it will be created with a set of novel and exciting applications and technological trends that will shape its performance targets while drastically redefining the core $5 \mathrm{G}$ services.

At the time when $6 \mathrm{G}$ systems will be made commercially available there will be further performance enhancements and new use cases added in the applications and services. To differentiate between the services provided by $5 \mathrm{G}$ and $6 \mathrm{G}$ there are three popular 5G use cases that will be provided as evolutionary examples. These are haptic communication for virtual and augmented reality (VR/AR), massive IoT integrated Smart City, and automation as well as manufacturing ( $\mathrm{Hu}$ et al. 2020, Chen et al. 2018).

- Haptic communication improves the touch sense of the outdated audiovisual communication over the Internet and is the key to unravel the prospective of VR/AR. This is of importance because a process of medical remote surgery requires ultra-sensitive delay of less than $1 \mathrm{~ms}$ which is not yet available in upcoming systems (Bastug et al. 2017, Elbamby et al. 2018, Park and Bennis 2018). 
- In the 5G system IoT has been implemented to make smart city a reality. In a smart city the major constituents such as public utilities, healthcare and monitoring are exclusively clever and considered discretely. 6G will adopt the universal approach in a fundamental way for a Smart city as compared to $5 \mathrm{G}$.

- In the industrial sector for any country it is vital to incorporate large number of robots into automation and transportation. These evolving concepts enhance the Industry 4.0 by exploiting mobile, analytics and cloud. 6G will fully support the industrial sector with massive IoT and AI capabilities.

6G will transport massive data with advanced computation capability and in this context AI (artificial intelligence) techniques will be implemented in all applications and services. Latest research has also geared towards edge cycling Fog radio access networking (Fog-RAN) and it has been shown that contents can be brought closer to user equipment (UEs), allowing for significant reduction in latency and power consumption.

\section{Conclusions}

The $5 \mathrm{G}$ system has been successfully standardized and it is being deployed worldwide. So, it is time to move on. The $6 \mathrm{G}$ infrastructure should fill the gap between 2020+ societal and business demands and what 5G can support. Research on $6 \mathrm{G}$ is in the phase of study. We study new performance targets beyond $5 \mathrm{G}$ in two stages: $5 \mathrm{G}+$ evolution and new $6 \mathrm{G}$ step. We learnt from the study three technology milestones for the upcoming years and these are described as follows:

- Short-term evolution. The deployment of 5G network deployment on a worldwide basis continues and all applications and services are available in large cities. To exploit the 5G evolution many vertical solutions are being considered. The $6 \mathrm{G}$ community has started to collect feedback from the $5 \mathrm{G}$ one.

- Medium-term evolution. A common discussion platform for frequency bands is considered for $6 \mathrm{G}$ and the architecture as well as the core technologies for smart networks are being developed. There is increased share of satellite communications. 5G business and vertical segments are well developed. The core technologies for $6 \mathrm{G}$ are selected and tested (RF, mm-wave, THz, optical). Also, 6G will employ extensive and advanced signal processing algorithms and applied AI/ML are being evaluated and compared. Testing and evaluations of the component networks of $6 \mathrm{G}$ are being worked out. Integration work towards experimental $6 \mathrm{G}$ testbed is underway.

- Long-term evolution. Advanced technologies for smart networks (SN) are considered. Cybersecurity and AI/ML in SN and 6G verticals are being exploited. Advanced radio technologies and DSP techniques are considered 
in 6G. Land and satellite networks are being integrated. Companies and business size in the field are increased significantly.

To justify our vision of future $5 \mathrm{G}+$ networks, we point out the need for closer connection of academia, standardizing bodies, industrial organizations and governments in demand-oriented roadmap. Also, the advancement of wireless communications is highly restricted by basic sciences, especially mathematics and physics. The contribution strategy is gradual evolution and performance enhancement mechanisms based on investment in more spectral and hardware/ software resources of mobile communications networks.

\section{References}

3GPP Technical Specification Group Services and System Aspects (2020) Technical Report TR 21.917, Release 17 description; Summary of Rel-17 Work Items (Release 17).

Andrews JG, Buzzi S, Choi W, Hanly SV, Lozano A, Soong ACK et al. (2014) What will 5G be? IEEE Journal on Selected Areas in Communications 32(Jun): 1065-1082.

Bastug E, Bennis M, Medard M, Debbah M (2017) Toward interconnected virtual reality: opportunities, challenges, and enablers. IEEE Communications Magazine 55(6): 110-117.

Bojkovic Z, Milovanovic D, Fowdur D (eds) (2020) $5 G$ Multimedia communication: technology, multiservices, and deployment. USA: CRC Press.

Chen M, Saad W, Yin C (2018) Virtual reality over wireless networks: quality-of-service model and learning-based resource management. IEEE Transactions on Communication 66(11): 5621-5635.

Dang S, Amin O, Shihada B, Alouini M-S (2020) What should 6G be? Nature Electronics 3(Jan): 20-29.

David K, Berndt H (2018) 6G Vision and requirements: is there any need for beyond 5G? IEEE Vehicular Technology Magazine 3(3): 72-80.

Elbamby MS, Perfecto P, Bennis M, Doppler K (2018) Toward low-latency and ultrareliable virtual reality. IEEE Network 32(2): 78-84.

FG-Net-2030 (2019) Blueprint of technology, applications and market drivers towards the year 2030 and beyond. ITU.

Hu F, Deng Y, Saad W, Bennis M, Aghvami AH (2020) Cellular-connected wireless virtual reality: requirements, challenges, and solutions. IEEE Communications Magazine 58(5): 105-111.

Milovanovic D, Bojkovic Z (2019) Optimal deployment of 5G network slicing based on complex network theory. In Proceedings of the International Conference TINKOS'19 (Belgrade), Serbia, 1-2.

Milovanovic D, Pantovic V, Bojkovic N, Bojkovic Z (2019) Advanced human centric 5GIoT in a smart city: requirements and challenges. In Proceedings of the International Conference HCC'19 11956, 285-296.

Park J, Bennis M (2018) URLLC-eMBB slicing to support VR multimodal perceptions over wireless cellular systems. In Proceedings of the International Conference GLOBECOM'18 (IEEE), 1-7.

Posio E (2020) 6GFlagship program. Oulu, Finland: University of Oulu. 
Saad W, Bennis M, Chen M (2020) A vision of 6G wireless systems: applications, trends, technologies, and open research problems. IEEE Network 34(3): 134-142.

Tripathi ND, Reed JH (2020) $5 G$ evolution - On the path to $6 G$ : Expanding the frontiers of wireless communications. Rohde \& Schwarz, 1-40. 
